\title{
Antecedentes de la reputación empresarial interna: el caso de una Cooperativa de Ahorro y Crédito colombiana
}

\author{
Mónica López-Santamaría ${ }^{1}$; ; Marietta Bucheli Gómez ${ }^{2}$; ;erlin Patricia Grueso Hinestroza ${ }^{3}$ y \\ Edison Jair Duque Oliva ${ }^{4}$
}

Recibido: 27 de septiembre de 2019 / Aceptado: 29 de septiembre de 2020 / Publicado: 15 de enero de 2021

Resumen. La construcción de la reputación empresarial comienza desde el interior de las organizaciones ya que, desarrollar una reputación interna favorable puede impulsar una dinámica de boca a boca positiva por parte de los grupos de interés internos. No obstante, aún existe escasa evidencia empírica respecto a la identificación de los factores determinantes de la reputación empresarial interna en el sector de la Economía Social y Solidaria. En consecuencia, este estudio tiene el propósito de identificar los antecedentes de la reputación empresarial desde la perspectiva de los grupos de interés internos en cuatro seccionales de una Cooperativa de Ahorro y Crédito colombiana. Para lograr el objetivo, se desarrolló un estudio cualitativo, en el que participaron siete grupos focales conformados por gerentes, trabajadores y miembros del Consejo de Administración. Para analizar la información obtenida se realizó un análisis de contenido de tipo temático en dos momentos y tres fases. Los resultados obtenidos muestran que los antecedentes de la reputación empresarial identificados por los participantes en el estudio son: 1) la calidad de los servicios prestados (oportunidad, servicio personalizado y calidez en la atención), 2) la cultura organizacional, relacionada con la dinámica familiar y los valores cooperativos que la fundamentan y 3) la apropiación de la identidad organizacional, que comprende la definición del perfil del asociado y el sentido de pertenencia. En las conclusiones se discuten los aspectos que hacen a las cooperativas diferentes de sus competidores y se plantean nuevas líneas de investigación.

Palabras clave: Calidad percibida del servicio; Cultura organizacional; Identidad organizacional; Reputación empresarial; Estrategia empresarial.

Claves Econlit: B55; L10; L14.

\section{[en] Antecedents of internal corporate reputation: The case of a Colombian Savings and Credit Cooperative}

\begin{abstract}
Building a corporate reputation begins from within organizations because when a business develops a favorable internal reputation can drive positive word of mouth dynamics from internal stakeholders. However, there is still little empirical evidence regarding the identification of the determining factors of internal corporate reputation in the Social and Solidarity Economy Sector. Therefore, this study aims to identify the antecedents of corporate reputation from the perspective of internal interest groups in four sections of a Colombian Savings and Credit Cooperative. To achieve the purpose, a qualitative study was carried out, in which seven focus groups made up of managers, workers and members of the Board of Directors participated. To analyze the information obtained, a thematic content analysis was carried out in two moments and three phases. The obtained results show that the antecedents of the corporate reputation identified by the participants in the study are: 1) the quality of the services provided (opportunity, personalized service and warmth of care), 2) the organizational culture, related to family dynamics and the cooperative values that underlie it and 3) the appropriation of organizational identity, which includes the definition of the associate's profile and the sense of belonging. The conclusions discuss the aspects that make cooperatives different from their competitors and propose new lines of research.
\end{abstract}

Keywords: Perceived quality of service; Organizational culture; Organizational identity; Corporate reputation; Business strategy.

Sumario. 1. Introducción. 2. Marco teórico. 3. Metodología. 4. Presentación de resultados. 5. Discusión de los resultados. 6. Conclusiones, límites de la investigación y futuras líneas de investigación. 7. Agradecimientos. 8. Referencias bibliográficas.

1 Universidad del Rosario, Bogotá-Colombia

Dirección de correo electrónico: monica.lopezs@urosario.edu.co.

2 Pontificia Universidad Javeriana, Bogotá-Colombia.

Dirección de correo electrónico: mbucheli@javeriana.edu.co.

3 Universidad del Rosario, Bogotá-Colombia.

Dirección de correo electrónico: merlin.grueso@urosario.edu.co.

4 Universidad Nacional de Colombia y Universidad Espíritu Santo, Ecuador.

Dirección de correo electrónico: ejduqueo@unal.edu.co. 
Cómo citar. López-Santamaría, M.; Bucheli Gómez, M.; Grueso Hinestroza, M.P.; Duque Oliva, E.J. (2021) Antecedentes de la reputación empresarial interna: el caso de una Cooperativa de Ahorro y Crédito colombiana. REVESCO. Revista de Estudios Cooperativos, vol. 137, e71865. https://dx.doi.org/10.5209/reve.71865.

\section{Introducción}

La gestión de los activos intangibles viene llamando la atención de quienes dirigen organizaciones en el sector de la Economía Social y Solidaria -ESS-, debido a que son considerados como un medio para favorecer el posicionamiento de estas (Schloderer, Sarstedt \& Ringle, 2014). Seguí-Mas y Server (2010a) indican que las cooperativas cuentan con intangibles de características particulares que necesitan ser estudiados para aportar información relevante, respecto a los procesos de toma de decisiones y la consecución de ventajas competitivas.

De acuerdo con Casimiro y Coelho (2015), la reputación empresarial es concebida como un recurso intangible que puede contribuir al fortalecimiento de las relaciones entre quienes hacen parte de cooperativas e impactar el rendimiento financiero de estas. De tal forma que, el estudio de la reputación en el sector de la ESS cobra relevancia porque permite, superar las dificultades de gestión de este intangible y enfrentar los desafíos de competitividad que actualmente se presentan en este sector (Casimiro \& Coelho, 2017).

En efecto, algunos autores indican que resulta necesario emprender estudios para comprender el comportamiento de la reputación empresarial en este sector de la economía (Castilla-Polo, SánchezHernández, Gallardo-Vázquez \& Ruíz, 2016), teniendo en cuenta que la reputación puede adoptar características específicas propias de las particularidades de este tipo de organizaciones, las cuales merecen ser estudiadas (Seguí-Mas \& Server, 2010b).

Particularmente en Colombia, de los 7,7 millones de asociados al sector de la ESS, el 82\% está asociado a cooperativas con actividad financiera siendo la mayoría de estas, Cooperativas de Ahorro y Crédito (Confecoop, 2018b). Durante la década de 1990, éstas organizaciones experimentaron una etapa de crisis comprometiendo su reputación en este país (Álvarez, 2016; Pardo-Martínez \& Huertas de Mora, 2014) y desencadenando un proceso posterior de preocupación por el posicionamiento de este tipo de cooperativas (Pardo \& Huertas, 2015).

Como consecuencia, dos décadas después, las perspectivas para el sector de la ESS planteadas en el marco del $18^{\circ}$ Congreso Nacional Cooperativo organizado por la Confederación de Cooperativas de Colombia -Confecoop- (2018a), indican que su apuesta fundamental está orientada a la configuración del "Pacto Nacional por la Economía Cooperativa y Solidaria" el cual, contempla dentro de sus principales metas la difusión y visibilidad del sector.

Por su parte, el Plan para una Década Cooperativa "Visión 2020" (Alianza Cooperativa Internacional ACI-, 2013:6) considera dentro de sus cinco temas críticos: "consolidar el mensaje cooperativista y definir la identidad de las cooperativas", teniendo en cuenta que, tal y como lo afirma la Organización Internacional del Trabajo -OIT- (2014:1) "el desarrollo de las cooperativas se encuentra con el obstáculo de la falta de conocimiento y comprensión del modelo de la empresa cooperativa".

Ahora bien, incrementar el conocimiento público sobre las cooperativas y su aporte al desarrollo sostenible, requiere de la participación de los grupos de interés involucrados directamente con el movimiento cooperativo (Organización de las Naciones Unidas -ONU-, 2011). Así, algunos autores advierten que una buena reputación comienza desde adentro de la organización (Fombrun, Gardberg \& Sever, 2000; Men, 2015), puesto que una reputación interna favorable puede impulsar una dinámica de boca a boca positiva y comportamientos de apoyo por parte de los grupos de interés internos (Hong \& Yang, 2011; Men \& Stacks, 2013).

En ese mismo sentido, Olmedo-Cifuentes y Martinez-León (2014) destacan una brecha importante en el estudio sobre la reputación desde el punto de vista de los clientes internos, si se tiene en cuenta que los estudios sobre este constructo se han orientado a explorar primordialmente la percepción de los clientes externos. Adicionalmente Men (2014), señala la escasa investigación orientada a indagar sobre el contenido de la reputación interna, pese al papel estratégico que esta dimensión del constructo posee, comprendiendo que los grupos de interés internos pueden llegar a moldear las percepciones de los grupos externos (Helm, 2011).

La exploración de los recursos desde la perspectiva interna permite explicar en mayor medida la dinámica de competitividad en las cooperativas de ahorro y crédito (Escobar, Velandia \& Navarro, 2018; Moyano, Puig, Bruque, 2008), si se considera que el énfasis de la gestión en este tipo de organizaciones está centrado en los grupos de interés internos. De esta manera, Bruque, Hernández, Vargas y Moyano (2002) destacan que el éxito competitivo de las cooperativas está relacionado en alguna medida con el grado de compromiso que tejen los asociados con la organización.

Así las cosas, identificar los elementos que anteceden a la reputación empresarial podría contribuir a precisar las actividades organizacionales en las que se debe invertir para gestionarla (Martínez y Rodríguez 
del Bosque, 2013), a la vez que permite a los investigadores asesorar a las empresas para mantener una ventaja competitiva por medio de la gestión efectiva de su reputación (Maurya, Kumar \& Mishra, 2017).

Este estudio tiene el propósito de identificar cuáles son los antecedentes de la reputación empresarial, en los que coinciden los grupos de interés internos de cuatro seccionales de una Cooperativa de Ahorro y Crédito colombiana. Para alcanzar dicho propósito se propone una aproximación cualitativa, dado que algunos autores (Clardy, 2012; Fombrun, 2012) advierten sobre la necesidad de favorecer estudios de naturaleza descriptiva, que reconozcan en mayor detalle las particularidades de la reputación empresarial, dependiendo del sector de la economía que se explore.

El artículo se ha organizado de la siguiente manera: en primer lugar, se desarrollan los conceptos que constituyen el objetivo del estudio. En segundo lugar, se describe la metodología que fue utilizada para identificar los antecedentes de la reputación empresarial, en los que coinciden los grupos de interés internos. En tercer lugar, se exponen los resultados obtenidos y se discuten a la luz de los autores e investigaciones revisadas. Finalmente se presentan las conclusiones, las limitaciones del estudio y las futuras líneas de investigación, así como los agradecimientos y las referencias bibliográficas.

\section{Marco teórico}

\subsection{Reputación empresarial y reputación interna}

De acuerdo con Fombrun (1996), la reputación se entiende como un recurso que requiere de tiempo para ser construido, puede percibirse de forma diferente según cada grupo de interés y es un atributo que permite determinar el lugar ocupado por la empresa respecto a sus rivales. Posteriormente, Fombrun (2012) precisa que este constructo se fundamenta en la percepción, implica un juicio colectivo que parte de los grupos de interés y se evalúa desde una perspectiva comparada.

Siguiendo a Chun (2005), las definiciones de la reputación empresarial se organizan en tres escuelas de pensamiento. Una Escuela Evaluativa en la que predomina la referencia al valor financiero desde la perspectiva de los inversores o directivos de las empresas. Una Escuela Impresional, que privilegia la alusión a las valoraciones que hacen los grupos de interés externos acerca de las impresiones que tiene respecto a la empresa. Una Escuela Relacional, a partir de la cual se entiende a la reputación como producto de las apreciaciones de los grupos de interés internos y externos ${ }^{5}$.

La Escuela Relacional permite reconocer que la reputación empresarial puede ser explorada desde la perspectiva interna y externa. De acuerdo con Pérez y Rodríguez del Bosque (2014), la reputación interna se refiere a la percepción que los grupos de interés internos tienen sobre el contenido de la identidad y la imagen de la organización, ya que esta percepción también se basa en lo que estos grupos quieren transmitir al exterior sobre la empresa.

Por otro lado, la reputación externa se configura a partir de la percepción que los grupos de interés externos tienen sobre la imagen que la organización proyecta. En este sentido, algunos autores entienden la reputación empresarial como las creencias o interpretaciones colectivas que existen sobre la identidad y la imagen de una empresa (Rao, 1994; Rindova \& Kotha, 2001), dependiendo del grupo de interés que se indague.

De esta manera, Gotsi y Wilson (2001) proponen que la cercanía conceptual entre la reputación y otros constructos lleva a entender a la primera desde dos perspectivas. Por una parte, la perspectiva análoga que entiende a la reputación como sinónimo de otros constructos, por ejemplo, la identidad y la imagen corporativa. Por otra parte, la perspectiva diferenciada que establece distinciones entre estos constructos cercanos, aunque no desconoce que son conceptos relacionados entre sí.

Esta última perspectiva, a la cual se adhiere el presente estudio, es complementada por Barnett Jermier y Lafferty (2006), quienes contribuyen a concretar las diferencias entre los conceptos de identidad e imagen. La identidad, se refiere a las características permanentes y centrales de las organizaciones que permiten distinguirlas de las demás. La imagen, se concibe como la representación que los grupos de interés externos tienen sobre la organización y que se diferencia de la identidad porque puede ser mucho más influenciada por aspectos de orden externo. La reputación, en consecuencia, se entiende como los juicios que los grupos de interés de orden interno y externo emiten sobre las percepciones que tienen de la identidad y de la imagen de la organización.

Para explorar el contenido de las investigaciones publicadas en revistas indexadas sobre reputación interna, se llevó a cabo una búsqueda del término "Internal Reputation” en título, resumen y palabras claves

\footnotetext{
"En esta investigación se propone delimitar a los grupos de interés con base en su nivel y poder de decisión sobre aspectos que afecten directamente a la empresa, considerando grupos de interés internos a: empleados, directivos, accionistas, inversores y socios de alianzas; y grupos de interés externos a: clientes (consumidores y usuarios), proveedores, competidores, autoridades locales, provinciales y estatales, medios de comunicación, el entorno y la sociedad general" (Olmedo-Cifuentes \& Martínez, 2011:6).
} 
de las publicaciones disponibles en ISI Web of Science (WOS) y Scopus, teniendo en cuenta que según Vanhala, Peltonen, Sundqvist, Nummenmaa y Järvelin (2020) el primero, se constituye en un índice citacional frecuentemente utilizado en este tipo de ejercicios de revisión de la literatura y el segundo, complementa el proceso, ya que contiene revistas indexadas que no se encuentran en WOS.

Con el objetivo de afinar la búsqueda se indagó sobre las publicaciones con el mismo término en título, resumen y palabras clave de las publicaciones disponibles en la revista Corporate Reputation Review. Este proceso de revisión de la literatura permite respaldar la afirmación de Men (2014), cuando indica la escasa investigación orientada a explorar este constructo.

Las investigaciones identificadas (Helm, 2011; Kim, Kang, Lee \& Yang, 2019; Kim \& Lim, 2020; Kulik \& Perry, 2008; Lee, 2020; Men 2014; 2015; Men \& Stacks, 2013; Olmedo-Cifuentes \& Martínez-León, 2014; Olmedo-Cifuentes, Martínez-León \& Davies, 2014; Primadini \& Syaebani, 2017; Villafañe, 2017; Wæraas \& Dahle, 2020) se encaminan a estudiar este recurso desde la perspectiva de los empleados y la gerencia, en relación con otros constructos como la gestión de los recursos humanos, la responsabilidad social, el liderazgo, la comunicación y el compromiso. Todo lo anterior, desde la perspectiva de sectores económicos diferentes al de la ESS.

\subsection{Antecedentes de la reputación empresarial: modelos teóricos y empíricos}

Con el propósito de verificar la afirmación de Gabbioneta, Ravasi y Mazzola (2007), respecto a la escasa evidencia empírica orientada a la definición de los antecedentes de la reputación empresarial, se llevó a cabo una revisión sistemática de literatura en ISI Web of Science (WOS) y Scopus. La búsqueda se enfocó en los artículos de investigación publicados en revistas indexadas que, tuvieran en su título, resumen y/o palabras clave, los conceptos relacionados con "antecedentes" y los términos asociados a "reputación empresarial" expresados ambos en idioma inglés.

Los resultados de la búsqueda arrojaron un total de quince artículos publicados acerca de este tema (Ali, Lynch, Melewar \& Jin 2015; Casimiro \& Coelho, 2015, 2017, 2019; Hormiga \& García-Almeida, 2016; Kim \& Kim, 2017; Martínez \& Rodríguez del Bosque, 2013; Maurya et al., 2017; Men \& Stacks, 2013; Mkumbuzi, 2015; Rindova Williamson, Petkova \& Sever 2005; Ruiz. Esteban \& Gutiérrez, 2014; Ruiz, Garcia \& Revilla, 2016; Vila-López \& Küster-Boluda, 2013; Walsh, Mitchell, Jackson \& Beatty, 2009). Además, nueve investigaciones fueron recuperadas, a partir del proceso de referencias citadas ${ }^{6}$ (Castilla-Polo et al., 2016; Fombrun, 2012; Helm, 2005; Martinez \& Olmedo, 2009; Martínez, Olmedo \& Zapata, 2013; Olmedo-Cifuentes \& Martinez, 2011; Olmedo-Cifuentes \& Martínez-León, 2014; Olmedo-Cifuentes, Martínez-León \& Davies 2014; Schwaiger, 2004).

Del total de 24 investigaciones revisadas, veintiuna son de carácter empírico y tres (Ali et al., 2015; Fombrun, 2012; Vila-López \& Küster-Boluda, 2013) llevan a cabo una propuesta exclusivamente teórica. Los estudios permiten evidenciar que, aunque se han desarrollado ejercicios orientados a determinar los antecedentes de la reputación empresarial, éstos en su conjunto no ofrecen un panorama concluyente.

Por ejemplo, el mayor nivel de acuerdo se encuentra en la identificación de la satisfacción como antecedente de la reputación empresarial, reconocida en siete de las 24 investigaciones revisadas (Casimiro \& Coelho, 2015, 2017; Kim \& Kim, 2017; Maurya et al., 2017; Ruiz et al., 2014, Ruiz et al., 2016; Walsh et al., 2009). No obstante, se identifican contradicciones respecto a otros antecedentes, por ejemplo, unos estudios se refieren a la confianza como antecedente de la reputación empresarial (Kim \& Kim, 2017; Maurya et al., 2017; Ruiz et al., 2014; Walsh et al., 2009) y otros como resultado de la gestión efectiva de la reputación (Ali et al., 2015; Casimiro \& Coelho, 2015). Por otro lado, Fombrun (2012) concibe a la innovación como un antecedente de la reputación y Castilla-Polo et al., (2016) como mediador entre la responsabilidad social corporativa y la reputación empresarial.

Este proceso de pesquisa también permite determinar que solo cinco de las 24 investigaciones recuperadas son llevadas a cabo en el sector de la ESS (Casimiro \& Coelho, 2015, 2017, 2019; Castilla-Polo et al., 2016; Martinez et al., 2013), las cuales en su totalidad son desarrolladas en España. Lo anterior, a pesar de las referencias que indican la importancia de incursionar en investigaciones sobre la reputación empresarial en este sector de la economía (Casimiro \& Coelho, 2017; Castilla-Polo et al., 2016; Schloderer et al., 2014) y favorecer la exploración de los antecedentes de este constructo, especialmente en los países en desarrollo (Maurya et al., 2017).

Estas investigaciones favorecen la precisión de algunos antecedentes de la reputación empresarial en el sector de la ESS, como: comunicación (Casimiro \& Coelho, 2015; 2017; 2019), cultura organizacional (Casimiro \& Coelho, 2015; 2019), satisfacción (Casimiro \& Coelho, 2015; 2017), valor percibido (Casimiro \& Coelho, 2017), imagen corporativa (Casimiro \& Coelho, 2017), responsabilidad social corporativa 
(Castilla-Polo et al., 2016), calidad de la gestión, innovación, gestión cultural de los recursos humanos y liderazgo (Martínez et al., 2013).

\subsection{Algunos apuntes sobre las cooperativas en Colombia}

De acuerdo con Ruiz et al (2014), es importante que los estudios sobre reputación empresarial reconozcan las particularidades del contexto en el que se va a llevar a cabo la investigación. En el mismo sentido, Chaves, Monzón, Pérez de Uralde y Radrigán (2013) señalan que la concepción de cooperativa se adapta a cada realidad nacional y se expresa a partir de sus formas jurídicas.

En Colombia la primera legislación cooperativa se estableció en el año de 1931 denominada Ley 134, a partir de la cual se acuerdan las condiciones para la configuración de organizaciones cooperativas en el país (Pardo-Martínez \& Huertas de Mora, 2014). Posteriormente, se reglamentó la Ley 79 de 1988 que precisó un marco legal para el desarrollo del sector fundamentado en la doctrina cooperativa y permitió la introducción de otras formas empresariales como fondos de empleados y asociaciones mutuales. Después se configuró la Ley 454 de 1998, que le otorgó al sector la denominación de "Economía Solidaria", facilitando la introducción de otras formas organizacionales de carácter más comunitario (Álvarez \& Serrano, 2006).

Así, en Colombia la Ley define a la cooperativa como una:

empresa asociativa sin ánimo de lucro en la cual los trabajadores o los usuarios según el caso son simultáneamente los aportantes y los gestores de la empresa, creada con el objeto de producir o distribuir conjunta y eficientemente bienes o servicios para satisfacer las necesidades de sus asociados y de la comunidad en general (Ley 79 de 1988:3).

Siguiendo a Silva (2012) el movimiento cooperativo pretende otorgarle un sentido más social a la economía, por lo cual en este ámbito conceptos como competitividad y rentabilidad propios de la economía tradicional capitalista se dotan de un contenido de solidaridad, cooperación e inclusión.

Otro de los aspectos diferenciales en el ámbito de la gestión de las cooperativas es que estas son autogestionadas por sus dueños, por lo tanto, sus miembros practican el autocontrol, el autogobierno y la autodirección porque actúan como usuarios, inversionistas y propietarios simultáneamente. La figura del gerente se integra a la dinámica de esta dirección colegiada y representa el puente entre el proceso estratégico y los demás procesos de carácter empresarial, convirtiéndose en el apoyo del equipo de asociados en materia de ejecución de las actividades de la cooperativa (Silva, 2012).

En consecuencia, la estructura de estas organizaciones se compone de una dimensión asociativa que se respalda en el contenido de la doctrina cooperativa y una dimensión empresarial que se encarga de los elementos de propiedad, gobierno y gestión, pero desde una perspectiva diferente a lo que acontece en el mercado de capitales (Silva, 2012).

Con relación a la configuración del sector en el contexto colombiano es posible decir que, de los 7,7 millones asociados al sector de la ESS, el 82\% está asociados a cooperativas (Confecoop, 2018b). Concretamente en Colombia "las cooperativas suman el 79.3\% del total de activos de la economía solidaria, el 90.2\% del patrimonio y el 86\% de los ingresos registrados en el año 2018" (Confecoop, 2018b: 64).

Para diciembre de 2019, el subsector de cooperativas con actividad financiera en Colombia está compuesto por 5 cooperativas financieras, un banco de naturaleza cooperativa, un banco de propiedad cooperativa y una compañía de financiamiento de propiedad cooperativa, las cuales están vigiladas por la Superintendencia Financiera. Además, 181 cooperativas autorizadas para ejercer la actividad financiera, las cuales están vigiladas por la Superintendencia de la Economía Solidaria y dentro de las que se encuentran las cooperativas de ahorro y crédito (Confecoop, 2019a). Las cooperativas con actividad financiera en Colombia cuentan con 3.844.506 asociados, por lo tanto, el impacto de estas organizaciones puede extenderse a 15.294 millones de personas, si se considera el ámbito familiar.

El subsector de las cooperativas con actividad financiera mostró un crecimiento importante en indicadores como activos, cartera bruta, depósitos y patrimonio para finales del año 2019, particularmente en diciembre de este año, la participación de este subsector se destacó en modalidades como el consumo y microcrédito con $9.33 \%$ y $9.97 \%$ respectivamente. No obstante, estas organizaciones pierden terreno en carteras de tradición como las carteras de consumo, debido a la competencia relacionada con bajas tasas de interés que ofrecen las entidades del sistema financiero tradicional (Confecoop, 2019a).

Con relación a las proyecciones de este subsector, se encuentra cómo frente a la Ley de Financiamiento, que modifica la tarifa general de impuesto de renta, y establece una sobretasa temporal para las entidades financieras en Colombia (4\% adicional para 2019, 3\% para 2020 y 3\% en 2021), se configura una oportunidad para las cooperativas de ahorro y crédito, ya que estas no se ven afectadas por la sobretasa de renta, lo cual les permite ofrecer tasas de intereses más favorables durante los próximos 3 años (Confecoop, 2019b). Lo anterior cobra relevancia si se tiene en cuenta que el sector de la ESS en Colombia, se plantea una ampliación de la base de asociados proyectada a 10 millones para el año 2025 (Acero, 2019). 


\section{Metodología}

\subsection{Aproximación}

Este estudio se desarrolla desde una aproximación cualitativa que, según Denzin y Lincoln (2005), implica que el investigador debe estudiar los fenómenos en su entorno natural e interpretarlos partiendo del reconocimiento de los significados que los informantes tienen de estos. Clardy (2012) señala la necesidad de complementar las aproximaciones cuantitativas alrededor del estudio de la reputación empresarial con ejercicios de naturaleza más descriptiva que permitan reconocer sus particularidades. En la misma línea, Fombrun (2012) indica que la aproximación cuantitativa no es la única alternativa para contribuir a la comprensión de la reputación empresarial, por lo que se requiere considerar otros caminos metodológicos.

\subsection{Diseño}

La investigación se enmarca en un diseño de estudio de caso simple con más de una unidad de observación (Yin, 2018), en este caso, representadas en cuatro seccionales de la Cooperativa de Ahorro y Crédito explorada. Este diseño de investigación se precisa como uno de los indicados para avanzar en la comprensión de las particularidades que las organizaciones cooperativas suponen en materia de gestión (Dávila, 2004).

\subsection{Unidad de observación}

El foco de este estudio es la exploración de una Cooperativa de Ahorro y Crédito con 58 años de funcionamiento, que se consolida como la segunda de este subsector en activos y cartera colocada a nivel nacional (Superintendencia de la Economía Solidaria, 2019). Además, según Informe de Confecoop (2019a) dicha organización se encuentra dentro de las veinte primeras cooperativas con actividad financiera en Colombia, teniendo en cuenta el volumen de cartera bruta.

De forma específica, se exploran cuatro seccionales ubicadas en la zona suroccidente de Colombia (Popayán, Cali, Armenia, Manizales), ya que según Confecoop (2018b) estas ciudades hacen parte de la segunda y tercera zona con mayor participación en activos del sector.

Durante ocho años de ejecución del plan estratégico de la organización analizada (2010-2020) se aprecia un crecimiento del $151 \%$ del activo, soportado en la cartera de crédito que ha alcanzado un crecimiento del $156 \%$. Así mismo, la cooperativa registra un incremento del $161 \%$ en los excedentes anuales durante el período de tiempo indicado y la inversión social alcanza los 8.796 millones con un crecimiento del $137 \%$ desde 2010. Con relación a la base social, se reporta un crecimiento del $45 \%$, por lo tanto, muestra un cierre a 2018 con 33.270 asociados de los 3.663.645 asociados a cooperativas con actividad financiera en Colombia (Cooperativa de Ahorro y Crédito, 2018).

\subsection{Informantes}

Los informantes están representados en 1) los socios miembros del Consejo de Administración, 2) los gerentes y 3) trabajadores de la cooperativa explorada, específicamente cajeros y promotores de servicios, los cuales se encuentran asociados a la cooperativa desde el momento de vinculación a esta.

Esta distribución se realizó teniendo en cuenta a Bastidas (2004), para quien estos actores organizacionales constituyen los tres sistemas que conforman la estructura de las organizaciones cooperativas. Los primeros que representan el sistema estratégico decisional, conformado por la Asamblea de Asociados y representada en el Consejo de Administración. Los segundos que configuran el sistema gerencial, los cuales actúan como una bisagra entre el sistema estratégico gerencial y el sistema operativo. Finalmente, los terceros que componen el sistema operativo conformado por los trabajadores, quienes llevan a cabo las tareas de naturaleza ejecutiva.

De la misma manera, para Matuleviciene y Stravinskiene (2015) los trabajadores y los socios son considerados como actores organizacionales principales en el estudio de la reputación empresarial y Rindova et al (2005) advierten que para indagar sobre la reputación empresarial resulta importante que los informantes posean conocimiento sobre el comportamiento de la empresa.

Como otro elemento de justificación para la elección de los informantes se encuentra que las cinco investigaciones revisadas sobre los antecedentes de la reputación empresarial en el sector de la ESS se centran en indagar un solo grupo de interés: trabajadores (Casimiro y Coelho, 2019; Martínez et al., 2013), gerentes (Castilla-Polo et al., 2016), asociados (Casimiro y Coelho, 2015; 2017), por lo que resulta importante emprender investigaciones que indaguen varios de estos grupos a la vez, con el propósito de explorar una visión común de las partes interesadas internas. 


\subsection{Técnicas de recolección de información}

Se implementan grupos focales, los cuales favorecen las interacciones de un grupo de personas que comparten ciertas características alrededor de un tema investigado (Saunders, Lewis y Thornill, 2009). Con relación a la exploración de los antecedentes de la reputación empresarial, Fombrun (2012) señala que estos se pueden indagar a partir de las evaluaciones colectivas que los grupos de interés hacen sobre el estado de la reputación en una empresa. De esta manera, se llevaron a cabo siete grupos focales entre julio y octubre de año 2018 con la participación de socios miembros del Consejo de Administración, gerentes y trabajadores de cuatro seccionales ubicadas en la zona suroccidente de Colombia, específicamente en las ciudades de Popayán, Cali, Armenia y Manizales 7 .

\subsection{Técnicas de análisis de la información}

Posterior a la implementación de los grupos focales se llevó a cabo la transcripción de las diez horas de grabación que estos representaron. Seguidamente se empleó como técnica de análisis de información el análisis de contenido de tipo temático que según, Braun y Clarke (2006: 79) "es un método para identificar, analizando y reportando patrones (temas) dentro de los datos". Este proceso se realizó a partir de un método de codificación descriptiva, el cual consiste en resumir en una palabra o frase corta el tema básico de un trozo de texto (Saldaña, 2013).

Se empleó una creación deductiva de códigos (Arbeláez \& Onrubia, 2014), que implica definir el nombre de las categorías, partiendo de lo que la teoría sugiere. Para tal efecto, las categorías se nombran teniendo como referencia las investigaciones sobre antecedentes de la reputación previamente revisadas, desde las cuales se construyó un diccionario de códigos con el propósito de precisar las categorías bajo las cuales se va a clasificar la información recolectada ${ }^{8}$. Sin embargo, resulta importante mencionar que, la creación deductiva de códigos no excluye la posibilidad de dejar emerger categorías cuando la recurrencia de los temas lo exige.

El proceso de codificación consideró dos momentos y tres fases. En el primer momento, se llevó a cabo la codificación de primer orden o codificación abierta, la cual según Charmaz (2006: 46) tiene como objetivo "permanecer abierto a todas las posibles indicaciones teóricas, a partir de la lectura de los datos". Luego se realizó la codificación de segundo orden o codificación enfocada, que según Saldaña (2013) busca los códigos iniciales más significativos o frecuentes para identificar categorías emergentes y agrupa los códigos que sean similares con el objetivo de sintetizar el número de categorías.

La primera y segunda fase de codificación fue llevada a cabo de forma independiente por dos investigadores; la tercera fase se configuró, a partir del encuentro de estos y la puesta en común de sus codificaciones de segundo orden, lo que permitió determinar el mapa definitivo de categorías. Después de la transcripción literal de los grupos focales, la totalidad del proceso de codificación se llevó a cabo con el apoyo del software licenciado de procesamiento de datos cualitativos Nvivo 12 Plus.

\section{Presentación de resultados}

Para el desarrollo de este apartado se lleva a cabo la presentación de los datos sociodemográficos de los informantes, la puesta en común de los antecedentes de la reputación empresarial en los que coincidieron los grupos de interés internos indagados y finalmente, se comentan las referencias que los informantes hacen de cada uno de los antecedentes.

La tabla 1 describe las características sociodemográficas de los grupos indagados y la tabla 2 el tiempo de vinculación a la cooperativa y al sector.

Tabla. 1. Datos sociodemográficos

\begin{tabular}{|c|c|c|c|c|c|c|}
\hline \multirow{2}{*}{ Grupo de interés } & \multicolumn{2}{|c|}{ Género } & \multicolumn{4}{c|}{ Nivel educativo } \\
\cline { 2 - 7 } & Femenino & Masculino & Técnico & Tecnológico & Pregrado & Postgrado \\
\hline Trabajadores: 10 & 7 & 3 & 1 & 2 & 4 & 3 \\
\hline $\begin{array}{c}\text { Consejos de } \\
\text { Administración: } 46\end{array}$ & 27 & 19 & 1 & 1 & 13 & 31 \\
\hline
\end{tabular}

Fuente: elaborado por los autores, a partir de los grupos focales realizados en Popayán (junio 2018), Cali (junio, 2018), Armenia (agosto, 2018) y Manizales (septiembre, 2018).

\footnotetext{
Ver guion del grupo focal en: https://padlet.com/investigacionreputacion/5b94ca5d0s4k.
}

Ver diccionario de códigos en: https://padlet.com/investigacionreputacion/5b94ca5d0s $4 \mathrm{k}$ 
En total se indagaron 10 trabajadores y 46 miembros del Consejo de Administración de los cuales, 34 son mujeres y 22 hombres. Con relación al nivel educativo de los informantes se reportan 2 técnicos, 3 con formación tecnológica, 17 con pregrado y 34 con formación de posgrado, lo cual se asocia con el perfil de asociados que admite la cooperativa explorada.

Tabla. 2. Tiempo de vinculación a la cooperativa y al sector de la ESS

\begin{tabular}{|c|c|c|c|c|c|}
\hline \multicolumn{6}{|c|}{ Trabajadores } \\
\hline \multicolumn{6}{|c|}{ Tiempo que lleva vinculado al sector } \\
\hline Menos de un año & 1 a 3 años & 3 a 5 años & 5 a 7 años & 7 a 10 años & Más de 10 años \\
\hline 3 & 3 & 2 & 1 & & 1 \\
\hline \multicolumn{6}{|c|}{ Tiempo que lleva vinculado a la cooperativa } \\
\hline Menos de un año & 1 a 3 años & 3 a 5 años & 5 a 7 años & 7 a 10 años & Más de 10 años \\
\hline 5 & 2 & 1 & 1 & & 1 \\
\hline \multicolumn{6}{|c|}{ Miembros del Consejo de Administración } \\
\hline \multicolumn{6}{|c|}{ Tiempo que lleva vinculado al sector } \\
\hline Menos de un año & 1 a 3 años & 3 a 5 años & 5 a 7 años & 7 a 10 años & Más de 10 años \\
\hline & 2 & 5 & 3 & 8 & 28 \\
\hline \multicolumn{6}{|c|}{ Tiempo que lleva vinculado a la cooperativa } \\
\hline Menos de un año & 1 a 3 años & 3 a 5 años & 5 a 7 años & 7 a 10 años & Más de 10 años \\
\hline 1 & 1 & 5 & 4 & 9 & 26 \\
\hline
\end{tabular}

Fuente: elaborado por los autores, a partir de los grupos focales realizados en Popayán (junio 2018), Cali (junio, 2018), Armenia (agosto, 2018) y Manizales (septiembre, 2018).

En la tabla 2 se evidencia que la mayor parte de los trabajadores reportan estar vinculados al sector y a la cooperativa hace menos de cinco años. Por otro lado, la mayoría de los miembros del Consejo de Administración reportan un período de tiempo de vinculación superior a siete años (36 personas al sector y 35 personas a la cooperativa), lo cual puede ser un indicador de que este último grupo de interés está conformado por personas que conocen del sector de la economía y de la cooperativa a la que se encuentran asociados.

Después de llevar a cabo la sistematización de la información recogida en los grupos focales y codificarla, teniendo en cuenta el diccionario de códigos, es posible precisar que los antecedentes de la reputación empresarial en los que coinciden los grupos de interés indagados son: calidad percibida del servicio, cultura organizacional e identidad organizacional. Lo anterior, considerando que estos son los antecedentes a los que se hizo referencia en la totalidad de los siete grupos focales realizados y los que cuentan con mayor número de afirmaciones que los respaldan'.

Con relación a la calidad percibida del servicio se encuentra que los grupos de interés indagados coinciden en referirse a este antecedente, resaltando las características de oportunidad en el servicio, atención personalizada y calidez en la atención.

Respecto a la oportunidad en el servicio, los trabajadores la conciben como una manera de destacarse frente a las entidades financieras tradicionales "acá siempre van a encontrar la oportunidad en la atención. Usted va a un banco y nadie se conoce con nadie, haga la cola y espere que lo atienda" (Trabajadora 4 Cali) y los miembros del Consejo de Administración como una estrategia de fidelización para los asociados "otra fortaleza es que si uno ha sido buen asociado los créditos son rápidos, es decir la oportunidad en los trámites y procesos" (Asociada 7 Armenia).

Otra característica mencionada por los grupos de interés indagados, específicamente por los trabajadores respecto a la calidad percibida del servicio, fue la atención personalizada que se refleja en las actividades propias de la dinámica cooperativa "algo que le llama mucho la atención al asociado cuando se va a vincular es la importancia que se le da cuando se le hace la bienvenida" (Trabajadora 3 Cali).

9 Para leer las referencias manifestadas por los informantes, respecto a las categorías analizadas, ir a: https://padlet.com/investigacionreputacion/5b94ca5d0s4k. 
Por su parte, los miembros del Consejo de Administración se refieren a la atención personalizada como una dinámica a partir de la cual se sienten reconocidos, a diferencia de los que sucede en otras entidades financieras "algo muy importante y es que nos tienen en cuenta como personas, sí, usted llega a la cooperativa y te saludan formal y alegremente. A todos nos llaman por el nombre, y vaya usted a un banco y nadie lo conoce a uno, haga la cola y ya, ahora con el cuento del cajero yo no volví al banco" (Asociada 3 Popayán).

Los grupos de interés indagados también refieren que el servicio prestado se configura desde la calidez. Así, para los trabajadores esta característica se constituye como un valor agregado del servicio "vienen a la caja y se les ofrece café, pagan y se sientan ahi a tomarse su café. Ese es como un valor agregado" (Trabajadora 1 Popayán) y representa otra estrategia de diferenciación respecto a las entidades financieras tradicionales "aquí son como más profundas las relaciones con los asociados; más que en un banco, en un banco tú lo atiendes y ya, se va y listo" (Trabajadora 2 Armenia). Esto último es respaldado por los miembros del Consejo de Administración cuando validan la importancia de la calidez recibida en el servicio prestado, por parte de la cooperativa "la calidez en la atención hacia el asociado que es muy importante" (Asociado 3 Cali).

En contraste, el cumplimiento en la prestación del servicio fue un atributo referenciado por los trabajadores, pero no por los miembros del Consejo de Administración. Los trabajadores resaltan cómo la atención en tanto característica del servicio se constituye en una forma diferencial con relación a otras entidades similares "el cumplimiento es lo principal, el cumplimiento que les damos a los asociados que están actualmente con nosotros, eso nos caracteriza" (Trabajador 1 Armenia); además de constituirse en un medio que puede generar referencias positivas por parte de los asociados "nosotros acá le brindamos una buena atención a las personas y les cumplimos con lo que les ofrecemos, así mismo se va regando la voz" (Trabajadora 1 Armenia).

Respecto a la cultura organizacional se encuentra que los grupos de interés indagados coinciden en referirse a este antecedente, resaltando las características asociadas a la dinámica familiar propia de las relaciones que favorece la cooperativa y los valores asociados a este tipo de organizaciones.

De esta manera, los trabajadores reconocen que las dinámicas de relacionamiento propuestas desde la cooperativa pueden ser valoradas como familiares, lo cual también se consolida como un valor agregado respecto a los competidores "parte del plus que se tiene es contacto con la gente. Aqui la calidez humana que se maneja con el asociado es casi como familiar, cosa que no se ve en otras cooperativas" (Trabajadora 3 Cali). Por su parte, los miembros del Consejo de Administración también asocian la calidad de su relación con la cooperativa con las relaciones familiares "yo estoy en otra cooperativa, que antes funcionaba con familiaridad, pero después cambio su modalidad y ya eso se acabó, solamente permanezco ahí porque tengo unos intereses, pero si uno quiere retirase pues se retira y ya. En cambio, uno acá llega y uno se siente como en casa" (Asociado 4 Popayán).

Cuando los trabajadores se refieren a los valores que fundamentan las relaciones en la cooperativa, mencionan los valores asociados al cooperativismo "son como más cercanas las relaciones, más solidarias; más que en un banco" (Trabajadora 2 Armenia) y resaltan la cultura del ahorro como un valor en el que se destaca la organización con relación a otras cooperativas "el ahorro en el sector de las cooperativas, ósea hay cooperativas de cooperativas, pero somos como la cooperativa que más se le aporta también al ahorro" (Trabajadora 1 Popayán). Por su parte, los miembros del Consejo de Administración también hacen referencia a los valores que fundamentan las relaciones con los asociados y que son propios del modelo cooperativo "yo no sé cómo se le pueda llamar a esto, pero hemos notado que aquí hay equidad y justicia en el tratamiento del asociado, es decir, no existen preferencias" (Asociado 4 Armenia).

Con relación a la identidad organizacional, se encuentra que los grupos de interés indagados coinciden en referirse a este antecedente, resaltando las características asociadas a la importancia de la definición del perfil del asociado y el sentido de pertenencia.

Si bien los trabajadores reconocen en la definición del perfil del asociado una fortaleza "una fortaleza es la calidad de los asociados, la calidad del asociado que nosotros tenemos, es decir, hay un perfil definido y eso es positivo" (Trabajadora 1 Cali). Los miembros del Consejo de Administración advierten que esto puede representar una dificultad si no se tienen claros los compromisos que le implica al asociado su vinculación a la doctrina cooperativa "tenemos una dificultad en la cooperativa en términos del perfil del asociado. Sí, tenemos un problema con el perfil del asociado, porque es que aquí se llega a la cooperativa porque me van a prestar, pero no porque soy cooperativista" (Asociado 1 Manizales).

Los grupos de interés también coinciden en advertir que la falta de sentido de pertenencia por parte de los grupos de interés internos genera impactos negativos para la cooperativa, por ejemplo, en materia de reconocimiento "la falta de sentido de sentido de pertenencia a veces nos afecta porque es ese voz a voz, de lo que se habla, lo que se dice, lo que nos ayuda a ser reconocidos" (Trabajadora 1 Armenia) y participación democrática "algo que afecta es la falta de sentido de pertenencia de los asociados en la participación de los espacios democráticos, siempre somos los mismos" (Asociado 1 Cali). 
Acerca de la identidad organizacional, los miembros del Consejo de Administración también resaltan algunas diferencias con respecto al sector financiero tradicional, en materia de los intereses cooperativos que van más allá de lo financiero "pero como estamos en una cooperativa no solo nos debe importar la solidez financiera sino qué tanto se cumple la filosofia del modelo cooperativo" (Asociada 1 Armenia) y la respuesta a las necesidades de la comunidad en la que la cooperativa se desarrolla "en el manejo de la economía solidaria cuando se piensa la constitución de la entidad se determina hacia dónde va a estar dirigida la cooperativa porque ese segmento tiene unas necesidades y tiene unos objetivos comunes entonces dentro de la constitución se plantea quiénes son los que van a hacer parte de esa cooperativa, lo que es distinto en el sector financiero tradicional" (Asociado 5 Cali).

Después de indagar en los grupos de interés internos sobre los antecedentes asociados a la gestión de la reputación empresarial, estos coinciden en referenciar de forma más recurrente asuntos relacionados con la calidad del servicio, la cultura organizacional y la identidad organizacional y concuerdan en la mayoría de las características que resaltan de estos antecedentes. No obstante, se encuentran algunas particularidades por grupo de interés como consecuencia de que los trabajadores resaltan la importancia del cumplimiento en la prestación del servicio, mientras que los miembros del Consejo de Administración hacen referencia a la identidad organizacional, enfatizando en las diferencias propias del sector de la ESS frente al sector financiero tradicional.

\section{Discusión de los resultados}

En este apartado se discuten los hallazgos, a partir de la contrastación teórica y se evidencian los aportes realizados tomando en cuenta las brechas identificadas en torno al estudio de la reputación, la reputación interna y sus antecedentes en el contexto del sector de la ESS.

De acuerdo con Castilla-Polo et al (2016) resulta necesario emprender estudios para comprender el comportamiento de la reputación empresarial en el sector de la ESS. Además, se encuentran referencias orientadas a señalar la escasa evidencia empírica relacionada con el estudio de la reputación interna (Men, 2014; Olmedo-Cifuentes \& Martinez-León, 2014). Por su parte, Maurya et al (2017) señala la importancia de favorecer la exploración de los antecedentes de la reputación empresarial especialmente en países en desarrollo.

Este estudio permite entonces, precisar tres antecedentes de la reputación interna a partir de las coincidencias y recurrencias referenciadas por los grupos de interés internos de una cooperativa de ahorro y crédito en Colombia. Según Casimiro y Coelho (2017) aportar información acerca de las vías para gestionar la reputación en este tipo de organizaciones resulta relevante en la medida en la que podría contribuir a comprender los desafíos de competitividad que actualmente afronta el sector, el cual plantea, en el caso colombiano, una ampliación de la base de asociados proyectada a 10 millones para el año 2025 (Acero, 2019).

De este modo, realizar una exploración de los antecedentes de la reputación desde la perspectiva interna puede contribuir a ampliar el conocimiento y comprensión del modelo de la empresa cooperativa promovido desde la Alianza Cooperativa Internacional (2012), ya que el reconocimiento de los recursos que aportan a una reputación interna favorable puede impulsar una dinámica de boca a boca positivo por parte de los grupos de interés internos (Hong \& Yang, 2011; Men \& Stacks, 2013).

Por otro lado, conocer los elementos que anteceden a la reputación empresarial puede contribuir a identificar las acciones que sería necesario emprender para gestionarla efectivamente (Martínez y Rodríguez del Bosque, 2013), por lo tanto, este estudio permite identificar que una ruta para impactar la reputación empresarial de adentro hacia afuera debe enfocarse en la gestión de los recursos estratégicos representados en: la calidad del servicio, el contenido de la cultura organizacional y la identidad organizacional.

La calidad percibida del servicio no es considerada en las investigaciones revisadas sobre los antecedentes de la reputación en el sector de la ESS, sin embargo, otras investigaciones exploradas sí la mencionan como antecedente de este constructo (Rindova et al., 2005; Vila-López y Küster-Boluda, 2013). De esta manera, con la referencia a este antecedente por parte de los grupos de interés indagados, se entiende que la apuesta por difundir y visibilizar el sector en Colombia (Confecoop, 2018a) y particularmente, la estrategia adoptada por la cooperativa explorada debe considerar el fortalecimiento y exaltación de aquellas características que favorecen la diferencia en el servicio prestado, ante todo en la dinámica del servicio personalizado y la calidez en la atención.

La identificación de la cultura organizacional como uno de los principales antecedentes de la reputación empresarial en esta cooperativa, encuentra soporte en los hallazgos de las investigaciones sobre este asunto en el sector de la ESS en España (Casimiro \& Coelho, 2015; 2019). Para Seguí-Mas y Server (2010a), los valores que fundamentan las culturas de cooperativas de ahorro y crédito son intangibles con características particulares que necesitan seguir siendo estudiadas con el objetivo de gestionar sus ventajas competitivas. Así, con este estudio se precisa que la dinámica familiar a la que se asocia la cultura organizacional y los 
valores mencionados por los grupos de interés, definen otra ruta a partir de la cual sería posible impactar positivamente la reputación como una alternativa de generación de ventaja competitiva para la cooperativa.

En la revisión teórica realizada sobre los modelos de antecedentes de la reputación empresarial, solamente Fombrun (2012) hace referencia a la identidad organizacional ${ }^{10}$, partiendo de una propuesta teórica que analiza antecedentes y consecuentes de la reputación empresarial. El presente estudio permite evidenciar que, desde la perspectiva de los grupos de interés internos en la cooperativa explorada, la identidad organizacional efectivamente puede ser considerada como una vía para impactar la reputación de este tipo de organizaciones.

Según Fombrun (1996) explorar la reputación implica referirse a las características que permiten a una organización ser diferente a sus principales rivales, lo que explica el hecho de que la identidad organizacional sea el otro antecedente de la reputación al que los grupos de interés indagados hacen referencia con mayor recurrencia, ya que es la identidad organizacional la que permite a esta organización cooperativa diferenciarse de las demás organizaciones con las que rivaliza directamente en el mercado.

No obstante, Bastidas (2019) advierte que organizaciones como las cooperativas de ahorro y crédito son identificadas como solidarias, pero no necesariamente practican este valor porque adoptan el funcionamiento de un banco tradicional y sus asociados no atienden lo que sucede en su interior, lo cual constituye sin duda, un desafío constante para este tipo de organizaciones en términos de coherencia.

En consecuencia, los hallazgos permiten evidenciar que, aunque la cooperativa explorada cuenta con la definición de un perfil para la admisión de asociados, los miembros del Consejo de Administración advierten que resulta indispensable promover la vinculación real del asociado al contenido de la doctrina cooperativa para fortalecer el sentido de pertenencia y representar coherentemente al cooperativismo.

Pese a que este análisis de los antecedentes de la reputación empresarial se orientó a discutir sobre los recursos en los que coincidieron los grupos de interés indagados, tal y como lo afirma Fombrun (1996) la reputación puede percibirse de forma diferente según cada grupo de interés.

Como consecuencia de esta aproximación, el estudio encuentra algunas diferencias entre los actores indagados, relacionadas con las cualidades que les atribuyen a estos antecedentes. De esta manera, los trabajadores destacan la característica del cumplimiento en la calidad percibida del servicio y, por otro lado, los miembros del Consejo de Administración resaltan las diferencias con el sector financiero tradicional cuando se refieren a la identidad organizacional.

Retomando a Bastidas (2004), los trabajadores representan el sistema operativo o esfera tecno-operativa, que desempeñan las tareas cotidianas en una organización cooperativa, por lo tanto, es esperable que este grupo de interés le otorgue relevancia al indicador de cumplimiento cuando se refiere a las características del servicio que se presta, ya que estos actores se constituyen en los directos responsables de garantizar esta cualidad en la prestación del servicio a los asociados. Primadini y Syaebani (2017) advierten por su parte, que aún no está claro qué factores contribuyen exactamente a la reputación corporativa desde la perspectiva de los empleados, por lo tanto, este estudio permite precisar que, para los trabajadores indagados, el cumplimiento en la prestación del servicio es en una característica que les permite desde su labor, contribuir positivamente a este constructo.

Ahora bien, si se tiene en cuenta que los miembros del Consejo de Administración representan el sistema estratégico decisional (Bastidas, 2004), es consecuente que este grupo de interés destaque las diferencias con el sector financiero tradicional cuando de identidad organizacional se trata, ya que se espera que estos actores tengan identificadas las características de la organización que podrían constituirse en fuentes de ventaja competitiva.

Es importante señalar a este respecto que las organizaciones cooperativas se estructuran a partir de dos dimensiones: una dimensión social, ética y cultural y una dimensión económica y/o productiva (Dávila, 2004; Orrego y Arboleda, 2005). La primera, relacionada con los beneficios otorgados a los asociados y la segunda, relacionada con la gestión económica, técnica, productiva y financiera de la organización (PardoMartínez \& Huertas-de-Mora, 2014).

En el presente caso, es posible precisar que los antecedentes de la reputación interna identificados pertenecen a la primera dimensión, lo que está sugiriendo que es a través de la gestión efectiva de esta, que la cooperativa de ahorro y crédito explorada puede impactar de forma positiva en su reputación empresarial, diferenciarse de sus principales competidores, fidelizar a sus grupos de interés internos y ser referenciada positivamente.

Los hallazgos de este estudio sugieren que para impactar positivamente la reputación empresarial se requiere, tal y como lo plantea Monzón (2012), utilizar la doctrina cooperativa como cuota de diferenciación complementaria a la meta empresarial y procurar por el fortalecimiento de la identidad cooperativa para

10 La identidad organizacional se entiende como un producto autorreflexivo del contenido de la cultura organizacional (Hatch, 1993). Así, los patrones de pensamiento y comportamiento que caracterizan la cultura de una organización pueden ayudar a que sus miembros doten de sentido las características distintivas y duraderas que contribuyen a definir "quiénes somos como organización" (Ravasi, 2016). 
prevenir el isomorfismo organizativo, que lleva a este tipo de empresas cooperativas a disolverse en el sistema económico como un agente empresarial más.

Como una alternativa fundamentada en esta apuesta por la diferenciación Malo (2004: 18) advierte que "imitar sin desnaturalizarse es posible con innovaciones sociales". Al respecto Dávila (2002) señala que la estructura y la dinámica de la cooperativa son una innovación en sí misma, no obstante, este estudio permite precisar que innovar socialmente a través de la calidad del servicio que se presta al asociado, la cultura organizacional con la que se cuenta y la identidad organizacional que se divulga, se convierte en una de las apuestas estratégicas orientada al fortalecimiento de la reputación empresarial en la cooperativa explorada.

En concordancia con Seguí-Mas y Server (2010b) este estudio encuentra que los elementos asociados a la reputación empresarial adoptan características específicas propias de las particularidades de este tipo de organizaciones cooperativas y además, contribuye en el estudio de estas, ya que dichas peculiaridades merecen ser exploradas para aportar información relevante en los procesos de toma de decisiones y contribuir en los propósitos de consecución de ventajas competitivas por parte de las cooperativas de ahorro y crédito (Seguí-Mas \& Server, 2010a).

En este sentido, la aproximación cualitativa sugerida por algunos autores (Clardy, 2012; Fombrun, 2012) resulta pertinente porque permite no solo identificar los antecedentes de la reputación, sino también describirlos con el objetivo de proporcionar más información respecto a la manera cómo los conciben los grupos de interés.

\section{Conclusiones, límites de la investigación y futuras líneas de investigación}

Este estudio pretende identificar cuáles son los antecedentes de la reputación empresarial, en los que coinciden los grupos de interés internos, de cuatro seccionales de una Cooperativa de Ahorro y Crédito colombiana.

Teniendo en cuenta los resultados obtenidos, es posible precisar que los procesos hacia los que la cooperativa explorada necesita orientar sus esfuerzos para impactar positivamente su reputación de adentro hacia afuera son: calidad del servicio, cultura organizacional e identidad organizacional.

Los principales antecedentes de la reputación empresarial identificados por los grupos de interés internos de la cooperativa explorada se orientan a hacer referencia a los aspectos de la organización que la hacen diferente a sus principales competidores. Lo anterior indica que, para gestionar efectivamente la reputación de esta organización de adentro hacia afuera, resulta necesario enfocarse en resaltar las diferencias y ventajas que trae el hecho de pertenecer a este tipo de organizaciones cooperativas, es decir, gestionar de forma eficiente los elementos que componen su dimensión social. Tal y como lo plantean Álvarez y Serrano (2006; 198) "en definitiva, se trata de ganar eficiencia con base en la integración consciente de recursos, orientados a fortalecer la identidad solidaria".

En este sentido, aquellas acciones estratégicas que se esfuerzan por asemejar este tipo de empresas a las organizaciones del sector financiero tradicional no necesariamente estarían siendo suficientemente efectivas cuando de gestionar la reputación empresarial se trata, porque, siguiendo a Dávila (2002: 115), se "requiere superar una práctica común y es la de denominar a la organización cooperativa con los conceptos y términos propios de la empresa tradicional, como si la cooperativa no tuviera su propio carácter".

Pese a los hallazgos alcanzados resulta necesario indicar las limitaciones del estudio y futuras líneas de investigación que se configuran. Aunque Clardy (2012) advierte sobre la necesidad de complementar las aproximaciones cuantitativas alrededor del estudio de la reputación empresarial con ejercicios de naturaleza más descriptiva, se considera relevante para el desarrollo de la investigación presente, que esta aproximación cualitativa sea complementada con un estudio de carácter cuantitativo en el que se explore el comportamiento de los antecedentes identificados, respecto a su influencia en la reputación empresarial de la cooperativa estudiada, por medio del modelamiento de ecuaciones estructurales.

Además, se recomiendan las siguientes rutas de investigación: 1) extender esta exploración a un número representativo de organizaciones cooperativas en Colombia, en la región y en el mundo, para explorar estos antecedentes considerando variables moderadoras como los tipos de organizaciones cooperativas, la antigüedad de estas, el país, entre otras, 2) trascender el abordaje de los grupos de interés internos y explorar las referencias que los grupos de interés externos puedan hacer respecto a los antecedentes de la reputación empresarial de estas organizaciones cooperativas y 3) apuntar a la realización de un análisis de caso comparativo entre una cooperativa de ahorro y crédito y un banco tradicional para determinar las diferencias entre los antecedentes de la reputación empresarial identificados por sus grupos de interés. 


\section{Agradecimientos}

Agradecemos el apoyo del Magíster en Políticas Públicas Julián Andrés Maya Mejía en el proceso de codificación, que permitió llevar a cabo una tercera fase de acuerdo entre investigadores. Lo anterior, con el objetivo de garantizar los criterios de calidad y rigor considerados en el marco de las investigaciones cualitativas.

\section{Referencias bibliográficas}

Acero, C. (2019) Las cooperativas somos una respuesta a los desafíos de la economía, del trabajo y del consumo. Cooperativas de las Américas. Región de la Alianza Cooperativa Internacional. Disponible en: https://www.aciamericas.coop/Las-cooperativas-somos-una-respuesta-a-los-desafios-de-la-economia-del-trabajo.

Ali, R., Lynch, R., Melewar, T. C., \& Jin, Z. (2015) The moderating influences on the relationship of corporate reputation with its antecedents and consequences: A meta-analytic review. Journal of Business Research, Vol. 68, $\mathrm{N}^{\mathrm{o}}$ 1, pp. 1105-1117. DOI: https://doi.org/10.1016/j.jbusres.2014.10.013.

Alianza Cooperativa Internacional -ACI- (2013) Plan para una década cooperativa. Disponible en: http://www.aciamericas.coop/IMG/pdf/ica blueprint es.pdf.

Álvarez, J. F. (2016) Articulación de políticas públicas dirigidas a las cooperativas. Propuestas para el caso colombiano en el marco de la convivencia pacífica y la internacionalización (Tesis Doctoral). Instituto Superior de Ciencias Sociales y Políticas-Universidad de Lisboa. Disponible en: https://www.repository.utl.pt/handle/10400.5/13022.

Álvarez, J. F., \& Serrano, R. (2006) Estructuras de integración del cooperativismo y la economía solidaria en Colombia. Cayapa. Revista Venezolana de Economía Social, Vol. 6, $\mathrm{N}^{\circ}$ 11, pp. 184-201. Disponible en: http://bdigital.ula.ve/storage/pdf/cayapa/v6n11/articulo9.pdf.

Arbeláez, M., \& Onrubia, J. (2014) Análisis bibliométrico y de contenido. Dos metodologías complementarias para el análisis de la revista colombiana Educación y Cultura. Revista de Investigaciones UCM, Vol. 14, No 23, pp. 14-31. Disponible en: http://www.revistas.ucm.edu.co/ojs/index.php/revista/article/view/5.

Barnett, M. L., Jermier, J. M., \& Lafferty, B. A. (2006) Corporate reputation: The definitional landscape. Corporate Reputation Review, Vol. 9, № 1, pp. 26-38. DOI: 10.1057/palgrave.crr.1550012.

Bastidas, O. (2004) La especificidad cooperativa. Apuntes para un cooperativismo alternativo. Ponencia presentada en el VIII Seminario Internacional de UNIRCOOP. La identidad de la cooperativa en el corazón de su éxito: Presentación de herramientas de gestión. Sherbrooke, Canadá, 4 al 8 de octubre.

Bastidas, O. (2019) La identidad como guía de acción de las cooperativas. Venezuela: Editorial InterCoop.

Braun, V., \& Clarke, V. (2006) Using thematic analysis in Psychology. Qualitative Research in Psychology, Vol. 3, $\mathrm{N}^{\circ}$ 2, pp. 77-101. DOI: http://dx.doi.org/10.1191/1478088706qp063oa.

Bruque, S., Hernández, M. J., Vargas, A., \& Moyano, J. (2002) ¿Son más competitivas las sociedades cooperativas? Un análisis en el sector de distribución farmacéutica. CIRIEC-España, Revista de Economía Pública, Social y Cooperativa, $\mathrm{N}^{\mathrm{o}} 42$, pp. 131-157. Disponible en: https://www.redalyc.org/articulo.oa?id=17404206.

Casimiro, M. G., \& Coelho, A. (2015) El impacto de la reputación en el desempeño de la organización en la perspectiva de los miembros de las cooperativas. Esic Market Economics and Business Journal, Vol. 46, N 1, pp. 37-65. DOI: 10.7200/esicm.150.0461.1e.

Casimiro, M. G., \& Coelho, A. (2017) A causal relationship model linking corporate reputation and customer-based brand equity: A customer perspective. Academia Revista Latinoamericana de Administración, Vol. 30, $\mathrm{N}^{\circ}$ 2, pp. 249-268. DOI: https://doi.org/10.1108/ARLA-09-2015-0253.

Casimiro, M. G., \& Coelho, A. (2019) The antecedents of corporate reputation and image and their impacts on employee commitment and performance: The moderating role of CSR. Corporate Reputation Review, Vol. 22, $\mathrm{N}^{\mathrm{o}} 1$, pp. 10-25. DOI: https://doi.org/10.1057/s41299-018-0053-8.

Castilla-Polo, F., Sánchez-Hernández, M. I., Gallardo-Vázquez, D., \& Ruíz, M del C. (2016) Diseño de un modelo de reputación para cooperativas oleícolas. Revista de Contabilidad, Vol. 19, $\mathrm{N}^{\circ}$ 1, pp. 154-167. DOI: https://doi.org/10.1016/j.rcsar.2015.10.003.

Charmaz, K. (2006) Constructing grounded theory. A practical guide through qualitative analysis. (First edition). Thousand Oaks, California: Sage.

Chaves, R., Monzón, J. L., Pérez de Uralde, J. M., \& Radrigán, M. (2013) La economía social en clave internacional. Cuantificación, reconocimiento institucional y visibilidad social en Europa, Iberoamérica y Norte de África. REVESCO. Revista de Estudios Cooperativos, $\mathrm{N}^{\circ}$ 112, pp. 122-150. DOI: 10.5209/rev_REVE.2013.v112.43069.

Chun, R. (2005) Corporate reputation: Meaning and measurement. International Journal of Management Reviews, Vol. 7, $\mathrm{N}^{\mathrm{o}}$ 2, pp. 91-109. DOI: 10.1111/j.1468-2370.2005.00109.x.

Clardy, A. (2012) Organizational reputation: Issues in conceptualization and measurement. Corporate Reputation Review, Vol. 15, № 4, pp. 285-303. DOI: https://doi.org/10.1057/crr.2012.1. 
Confederación Colombiana de Cooperativas -Confecoop- (2018a) Apertura del $18^{\circ}$ Congreso Nacional Cooperativo: "Confianza y Acción”. Disponible en: https://confecoop.coop/actualidad/actualidad-2019/exitosa-apertura-del-18congreso-nacional-cooperativo-confianza-y-accion/.

Confederación Colombiana de Cooperativas -Confecoop- (2018b) Informe de desempeño 2018 “Confianza y Acción”. Disponible en: https://confecoop.coop/wp-content/uploads/2019/12/INFORME-2018.pdf.

Confederación Colombiana de Cooperativas -Confecoop- (2019a) Sector cooperativo con actividad financiera. Diciembre 2019. Unidad de Investigaciónes Económicas. Observatorio Cooperativo No. 55. Disponible en: https://confecoop.coop/wp-content/uploads/2020/03/No.-55-Sector-cooperativo-con-actividad-financieraDiciembre-2019.pdf.

Confederación Colombiana de Cooperativas -Confecoop- (2019b) Perspectivas para el cooperativismo 2019. Unidad de Investigaciones Económicas. Observatorio Cooperativo No. 48. Disponible en: https://confecoop.coop/wpcontent/uploads/2019/02/Observatorio-No-48.pdf.

Congreso de la República de Colombia. Ley 79 de 1988. Por la cual se actualiza la legislación cooperativa. Disponible en: https://www.funcionpublica.gov.co/eva/gestornormativo/norma.php?i=9211.

Cooperativa de Ahorro y Crédito (2018) Informe de gestión 2018. Disponible en: https://www.coasmedas.coop/images/INFORME_DE_GESTI\%C3\%93N_COASMEDAS_2018.pdf.

Dávila, R. (2002) Las cooperativas en Colombia: innovación organizacional y novedad académica. Cuadernos de

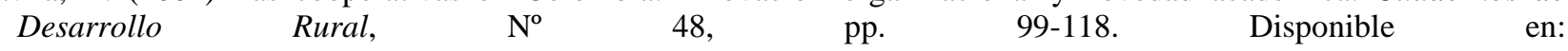
https://revistas.javeriana.edu.co/index.php/desarrolloRural/article/view/1996.

Dávila, R. (2004) Innovación y éxito en la gerencia cooperativa: casos exitosos de cooperativas rurales de ahorro y crédito. Bogotá-Colombia: Javergraf.

Denzin, N. K., \& Lincoln, Y. S. (Eds.). (2005) The SAGE Handbook of Qualitative Research (Third edition). Thousand Oaks, California: Sage.

Escobar, A., Velandia, G., \& Navarro, E. (2018) Gestión del conocimiento y competitividad en las cooperativas con sección de ahorro y crédito. REVESCO. Revista de Estudios Cooperativos, Primer Cuatrimestre, N $^{\circ} 127$, pp. 90115. DOI: http://dx.doi.org/10.5209/REVE.59769.

Fombrun, C. J. (1996) Reputation: Realising value from the corporate image. Boston: Harvard Business School Press.

Fombrun, C. J., Gardberg, N. A., \& Sever, J. M. (2000) The reputation quotient: A multi-stakeholder measure of corporate reputation. The Journal of Brand Management, Vol. 7, pp. 241-255. DOI: https://doi.org/10.1057/bm.2000.10.

Fombrun, Ch. J. (2012) The building blocks of corporate reputation: Definitions, antecedents, consequences. En: Barnett, M. L., \& Pollock, T. G (Eds.). The Oxford Handbook of Corporate Reputation. United Kingdom: Oxford University Press, pp. 115-134.

Gabbioneta, C., Ravasi, D., \& Mazzola, P. (2007) Exploring the drivers of corporate reputation: A study of italian securities analysis. Corporate Reputation Review, Vol. 10, No 2, pp. 99-123. DOI: 10.1057/palgrave.crr.1550048.

Gotsi, M., \& Wilson, A. M. (2001) Corporate reputation: Seeking a definition. Corporate Communications, Vol. 6, pp. 24-30. DOI: https://doi.org/10.1108/13563280110381189.

Hatch, M. J. (1993) The dynamics of organizational culture. Academy of Management Review, Vol. 18, No 4, pp. 657693. DOI: $10.2307 / 258594$.

Helm, S. (2005) Designing a formative measure for corporate reputation. Corporate Reputation Review, Vol. 8, No 2 , pp. 95-109. DOI: https://doi.org/10.1057/palgrave.crr.1540242.

Helm, S. (2011) Employees' awareness of their impact on corporate reputation. Journal of Business Research, Vol. 64, $\mathrm{N}^{\mathrm{o}}$ 7, pp. 657-663. DOI: https://doi.org/10.1016/j.jbusres.2010.09.001.

Hong, S. Y., \& Yang, S. U. (2011) Public engagement in supportive communication behaviors toward an organization: Effects of relational satisfaction and organizational reputation in public relations management. Journal of Public Relations Research, Vol. 23, № 2, pp. 191-217. DOI: 10.1080/1062726X.2011.555646.

Hormiga, E., \& García-Almeida, D. J. (2016) Accumulated knowledge and innovation as antecedents of reputation in new ventures. Journal of Small Business and Enterprise Development, Vol. 23, No 2, pp. 428-452. DOI: https://doi.org/10.1108/JSBED-03-2014-0038.

Kim, S. B., \& Kim, D. Y. (2017) Antecedents of corporate reputation in the hotel industry: The moderating role of transparency. Sustainability, Vol, 9, Nº, pp. 951-966. DOI: 10.3390/su9060951.

Kim, Y., \& Lim, H. (2020). Activating constructive employee behavioural responses in a crisis: Examining the effects of pre-crisis reputation and crisis communication strategies on employee voice behaviours. Journal of Contingencies and Crisis Management. Vol. 28, pp. 141-157- DOI: 10.1111/1468-5973.12289.

Kim, Y., Kang, M., Lee, E., \& Yang, S. U. (2019) Exploring crisis communication in the internal context of an organization: Examining moderated and mediated effects of employee-organization relationships on crisis outcomes. Public Relations Review, Vol. 45, No 3, pp. 101- 117. DOI: https://doi.org/10.1016/j.pubrev.2019.04.010.

Kulik, C. T., \& Perry, E. L. (2008) When less is more: The effect of devolution on HR's strategic role and construed image. Human Resource Management, Vol. 47, No 3, pp. 541-558. DOI: 10.1002/hrm.20231.

Lee, Y. (2020) Toward a communality with employees: The role of CSR types and internal reputation. Corporate Reputation Review, Vol. 23, No 1, pp. 13-23. DOI: https://doi.org/10.1057/s41299-019-00069-X.

Malo, M-C. (2004) La gestión estratégica de las cooperativas y de la asociación de la economía social. Documento 47. Disponible en: https://base.socioeco.org/docs/documento_47.pdf. 
Martínez, I. M., \& Olmedo, I. (2009) La medición de la reputación empresarial: problemática y propuesta. Investigaciones Europeas de Dirección y Economía de la Empresa, Vo. 15, No 2, pp. 127-142. DOI: https://doi.org/10.1016/S1135-2523(12)60093-8.

Martínez, I. M., Olmedo, I., \& Zapata, J. (2013) Reputación percibida por el profesorado de las cooperativas de educación: medición e influencia. CIRIEC-España, Revista de Economía Pública, Social y Cooperativa, Vol. 77, No 1, pp. 237-260. Disponible en: https://www.redalyc.org/articulo.oa?id=17429864010.

Martínez, P., \& Rodríguez del Bosque, I. (2013) Intellectual capital and relational capital: The role of sustainability in developing corporate reputation. Intangible Capital, Vol. $\quad 9, \quad \mathrm{~N}^{\mathrm{o}} \quad 1, \quad$ pp. $262-280$. https://www.intangiblecapital.org/index.php/ic/article/view/378.

Matuleviciene, M., \& Stravinskiene, J. (2015) The importance of stakeholders for corporate reputation. Engineering Economics, Vol. 26, № 1, pp. 75-83. DOI: http://dx.doi.org/10.5755/j01.ee.26.1.6921.

Maurya, U., Kumar, S. P., \& Mishra, P. (2017) An empirical examination of the dimensions, antecedents, and consequences of corporate reputation. International Journal of Applied Business and Economic Research, Vol. 15, $\mathrm{N}^{\circ} 6$, pp. $99-115$.

Men, L. R. (2014) Internal reputation management: The impact of authentic leadership and transparent communication. Corporate Reputation Review, Vol. 17, № 4, pp. 254-272. DOI: doi:10.1057/crr.2014.14.

Men, L. R. (2015) Employee engagement in relation to employee-organization relationships and internal reputation: Effects of leadership communication. Public Relations Journal, Vol. 9, $\mathrm{N}^{\mathrm{o}}$ 2, pp. 11-22. Disponible en: https://prjournal.instituteforpr.org/wp-content/uploads/2015v09n02Men.pdf.

Men, L. R., \& Stacks, D. W. (2013) The impact of leadership style and employee empowerment on perceived organizational reputation. Journal of Communication Management, Vol. 17, $\mathrm{N}^{\mathrm{o}}$ 2, pp. 171-192. DOI: https://doi.org/10.1108/13632541311318765.

Mkumbuzi, W. P. (2015) Corporate governance and intangibles disclosure as determinants of corporate reputation. Asian Social Science, Vol. 11, No 23, pp. 192-208. DOI: 10.5539/ass.v11n23p192.

Monzón, J. L. (2012) Las cooperativas ante la globalización: magnitudes, actividades y tendencias. EKONOMIAZ. Revista Vasca de Economía, Vol. 79, $\mathrm{N}^{\mathrm{o}}$ 1, pp. 13-30. Disponible en: https://dialnet.unirioja.es/servlet/articulo?codigo $=3876119$.

Moyano, J., Puig, F., \& Bruque, S. (2008) Los determinantes de la competitividad en las cooperativas. CIRIEC-España, Revista de Economía Pública, Social y Cooperativa, $\mathrm{N}^{\mathrm{o}}$ 61, pp. 233-249. Disponible en: https://www.redalyc.org/pdf/174/17412302011.pdf.

Olmedo-Cifuentes, I., \& Martínez, I. M. (2011). Medida de la reputación empresarial en pymes de servicios. European Journal of Management and Business Economics, Vol. 20, $\mathbf{N}^{\mathrm{o}}$ 3, pp. 77-121. Disponible en: https://www.redalyc.org/pdf/2741/274120373002.pdf.

Olmedo-Cifuentes, I., \& Martínez-León, I. M. (2014) Influence of management style on employee views of corporate reputation. Application to audit firms. BRQ Business Research Quarterly, Vol. 17, No 4, pp. 223-241. DOI: https://doi.org/10.1016/j.brq.2013.08.001.

Olmedo-Cifuentes, I., Martínez-León, I. M., \& Davies, G. (2014) Managing internal stakeholders' views of corporate reputation. Service Business, Vol. 8, No 1, pp. 83-111. DOI: https://doi.org/10.1007/s11628-013-0188-8.

Organización de las Naciones Unidad -ONU- (2011) Report of the expert group meeting cooperatives in social development: Beyond 2012. Disponible en: https://www.un.org/esa/socdev/egms/docs/2011/coops-egm-report.pdf.

Organización Internacional del Trabajo -OIT- (2014) La forma cooperativa de hacer negocios. Las cooperativas ayudan a asegurar medios de vida. Disponible en: http://www.relats.org/documentos/ESS.OIT.Actemp.pdf.

Orrego, C. I., \& Arboleda, O. L. A. (2005) Las organizaciones de economía solidaria: Un modelo de gestión innovador. Cuadernos de Administración, Vol. 21, N $\mathrm{N}^{\mathrm{O}}$ 34, pp. 97-110. Disponible en: https://base.socioeco.org/docs/_pdf_2250_225020897004.pdf.

Pardo, L. P., \& Huertas, M. V. (2015) Proceso histórico de las prácticas cooperativas en Colombia. En: Mutuberría, V., \& Plotinsky, D (Eds). La economía social y solidaria en la historia de América Latina y el Caribe. Cooperativismo, desarrollo comunitario y Estado (tomo II). Ciudad Autónoma de Buenos Aires: Ediciones Idelcoop (p. 153-168). Disponible https://www.idelcoop.org.ar/sites/www.idelcoop.org.ar/files/u15/congreso_de historia_y_economia_social_tomo_2.pdf.

Pardo-Martínez., L. P., \& Huertas de Mora, M. V. (2014) Tendencias en la gestión de empresas sin ánimo de lucro. Caso: cooperativas. Bogotá-Colombia: Universidad Cooperativa de Colombia. Disponible en: https://ediciones.ucc.edu.co/index.php/ucc/catalog/book/7.

Pérez, A., \& Rodríguez del Bosque, I. R. (2014) Identidad, imagen y reputación de la empresa: integración de propuestas teóricas para una gestión exitosa.Cuadernos de Gestión, Vol. 14, pp. 97-126. DOI: 10.5295/cdg.130389ap.

Primadini, D. V., \& Syaebani, M. I. (2017) Factors affecting employee's awareness of their impact on corporate reputation. Journal Ekonomi Dan Bisnis, Vol. 20, $\mathrm{N}^{\mathrm{o}}$ 2, pp. 215-228. Disponible en: https://pdfs.semanticscholar.org/cdb6/757b4b6eb36baf9f53f5e1234c31d094ba3b.pdf.

Rao, H. (1994) The social construction of reputation: Certification contests, legitimation, and the survival of organizations in the American automobile industry: 1985-1912. Strategic Management Journal, Vol. 15, pp. 29-44. DOI: https://www.jstor.org/stable/2486809. 
Ravasi, D. (2016) Organizational identity, culture, and image. In: Schultz, M., Ashforth, B.E \& Ravasi, D. (Eds.). The Oxford Handbook of Organizational Identity. (pp. 65-78). Oxford: Oxford University Press

Rindova, V. P., \& Kotha, S. (2001) Accumulating reputation through strategic action flows: Lessons from Amazon.com and its competitors in Internet retailing. University of Maryland, College Park, MD: Unpublished Paper.

Rindova, V. P., Williamson, I. O., Petkova, A. P., \& Sever, J. M. (2005) Being good or being known: An empirical examination of the dimensions, antecedents, and consequences of organizational reputation. Academy of Management Journal, Vol. 48, № 6, pp. 1033-1049. DOI: 10.2307/20159728.

Ruiz, B., Esteban, Á., \& Gutiérrez, S. (2014) Determinants of reputation of leading spanish financial institutions among their customers in a context of economic crisis. Business Research Quarterly, Vol. 17, No 1, pp. 2-20. DOI: https://doi.org/10.1016/j.brq.2014.04.002.

Ruiz, B., García, J. A., \& Revilla, A. J. (2016) Antecedents and consequences of bank reputation: A comparison of the United Kingdom and Spain. International Marketing Review, Vol. 33, $\mathrm{N}^{\mathrm{o}}$ 6, pp. 781-805. DOI: https://doi.org/10.1108/IMR-06-2015-0147.

Saldaña, J. (2013) The coding manual for qualitative researches. (Second edition). Thousand Oaks, California: SAGE Publications.

Saunders, M., Lewis, Ph., \& Thornill, A. (2009) Research methods for business students (Fifth edition). Italy: Pearson Education Limited.

Schloderer, M. P., Sarstedt, M., \& Ringle, C. M. (2014) The relevance of reputation in the nonprofit sector the moderating effect of socio-demographic characteristics. International Journal of Nonprofit and Voluntary Sector Marketing, Vol. 19, № 1, pp. 110-126. DOI: https://doi.org/10.1002/nvsm.1491.

Schwaiger, M. (2004) Components and parameters of corporate reputation an empirical study. Schmalenbach Business Review, Vol. 56, pp. 46-71. DOI: https://doi.org/10.1007/BF03396685.

Seguí-Mas, E., \& Server, R. J. (2010a) Caracterización del business capital de las cooperativas de crédito a través del análisis Delphi. REVESCO. Revista de Estudios Cooperativos, Tercer Cuatrimestre, N ${ }^{\circ}$ 103, pp. 101-122. Disponible en: https://revistas.ucm.es/index.php/REVE/article/view/REVE1010440101A.

Seguí-Mas, E., \& Server, R. J. (2010b) El capital relacional de las cooperativas de crédito en España: un estudio cualitativo de sus intangibles sociales mediante el análisis Delphi. REVESCO. Revista de Estudios Cooperativos,
Primer
Cuatrimestre,
101
pp.
107-131.
Disponible
en: https://revistas.ucm.es/index.php/REVE/article/view/REVE1010230107A.

Silva, A. M. (2012) Algunas pautas de gestión para la coopetitividad. En: J. F, Álvarez. (Ed). Cooperativismo e internacionalización (Tomo II). Condiciones y lineamientos para su desarrollo en Colombia (p. 161-176). Bogotá: Legis. Disponible en: http://www.socioeco.org/bdf fiche-document-3614 es.html.

Superintendencia de la economía solidaria (2019) Base de datos de organizaciones que reportan a la entidad a abril de 2019. Disponible en: http://www.supersolidaria.gov.co/es/entidades-vigiladas/entidades-vigiladas-que-reportaninformacion-2019.

Vanhala, M, Lu, C., Peltonen, J., Sundqvist, S., Nummenmaa, J., \& Järvelin, K. (2020) The usage of large data sets in online consumer behaviour: A bibliometric and computational text-mining-driven analysis of previous research. Journal of Business Research, Vol. 106, $\mathrm{N}^{\mathrm{o}}$ 1, pp. 46-59. DOI: https://doi.org/10.1016/j.jbusres.2019.09.009.

Vila-López, N., \& Küster-Boluda, I. (2013) Antecedentes de reputación corporativa en cadenas televisivas. Revista de Ciencias Sociales, Vol. 19, $\quad \mathrm{N}^{\mathrm{o}} 2, \quad$ pp. 334-348. Disponible en: https://dialnet.unirioja.es/servlet/articulo?codigo=4385163.

Villafañe, J. (2017) Claves empíricas de la satisfacción y del compromiso del talento en las organizaciones. El profesional de la información (EPI), Vol. 26, № 6, pp. 1159-1170. DOI: https://doi.org/10.3145/epi.2017.nov.15.

Wæraas, A., \& Dahle, D. Y. (2019) When reputation management is people management: Implications for employee voice. European Management Journal. Vol. 38, pp, 277-287. DOI: https://doi.org/10.1016/j.emj.2019.08.010.

Walsh, G., Mitchell, V. W., Jackson, P. R., \& Beatty, S. E. (2009) Examining the antecedents and consequences of corporate reputation: A customer perspective. British Journal of Management, Vol. 20, $\mathrm{N}^{\mathrm{o}}$ 1, pp. 187-203. DOI: 10.1057/palgrave.crr.1550048.

Yin, R, K. (2018) Case study research and applications. Design and methods. (Sixth Edition). Thousand Oaks, California: SAGE Publications. 